\title{
Análisis del proceso de extruido de un tubo de material compuesto en resina isoftalica y fibra de vidrio.
}

\begin{abstract}
Analysis of the extruded process of a tube with an isophthalic resin composite material in fiber and glass.
\end{abstract}

Edgar Fabián Sánchez Carrión. ${ }^{1}$, Edison Marcelo Castillo Cárdenas. ${ }^{2}$, Víctor David Bravo Morocho. ${ }^{3} \&$ Edison Patricio Abarca Pérez. ${ }^{4}$

\begin{abstract}
.
DOI: https://doi.org/10.33262/cienciadigital.v2i2.104

The present work analyzes an extrusion process for composite material, which is a process for obtaining profiles of constant section based on composite materials by means of fiberglass reinforcement and thermostable matrix. An experimental study of the process variables was established, in which each of the variables involved in the extrusion process were modified, such as fiber matting, the quantity of fiberglass yarns, the concentrations of initiators in the isfotalica resin (matrix), speed in the pullers, with which a product resulting from characteristics suitable to the process used is obtained which is compared with traditionally diffused materials.
\end{abstract}

Keywords: Composite Material, Fiber Reinforced Plastic (Frp), Fiberglass, Mathematical Model, Concurrent Design.

\section{Resumen.}

El presente trabajo se analiza un proceso de extrusión para material compuesto, que es un proceso para la obtención de perfiles de sección constante en base a materiales

\footnotetext{
${ }^{1}$ Escuela Superior Politécnica de Chimborazo, Chimborazo, Ecuador, esanchez_c@espoch.edu.ec

${ }^{2}$ Escuela Superior Politécnica de Chimborazo, Chimborazo, Ecuador, edison.castillo@espoch.edu.ec

${ }^{3}$ Escuela Superior Politécnica de Chimborazo, Chimborazo, Ecuador, victor.bravo@espoch.edu.ec

${ }^{4}$ Escuela Superior Politécnica de Chimborazo, Chimborazo, Ecuador, edison.abarca@espoch.edu.ec
} 
compuestos por medio refuerzo de fibra de vidrio y de matriz termoestable. Se estableció un estudio experimental de las variables del proceso, en el cual se modificó cada una de las variables que intervienen en el proceso de extrusión como son el entramado de la fibra mat, la cantidad de hilos de fibra de vidrio las concentraciones de iniciadores en la resina isfotalica (matriz), velocidad en los pullers, con lo que se obtiene un producto resultante de características adecuadas al proceso utilizado el cual es comparado con materiales tradicionalmente difundidos.

Palabras Claves: material compuesto, fiber reinforced plastic (FRP), fibra de vidrio, modelo matemático, diseño concurrente.

\section{Introducción.}

Un material compuesto está conformado por dos o más materiales que son diferentes entre sí, para lo cual existen diferentes métodos de fabricación. El proceso de extrusión también llamado pultrusión, de este tipo de materiales de matriz termoestable, se esquematiza en la figura $1 \mathrm{y}$ consiste en que fibras de vidrio pasen por un recipiente que contiene resina, en la cual las fibras se saturan y pasan por un conformador (molde) en el que se da la sección deseada todo este conjunto resina/fibra es tirado por un sistema hidráulico. Cuando la resina que está cargada de rellenos, acelerantes, agentes desmoldantes, pigmentos y aditivos pasa por el molde calefaccionado activando la polimerización de la resina (curado). Las resinas que se utilizan en este proceso de extrusión pueden ser de éster vinilo, epoxica o poliéster, pero aproximadamente el $90 \%$ es poliéster.

Figura 1. Proceso de extrusión (pultrusion) de material compuesto

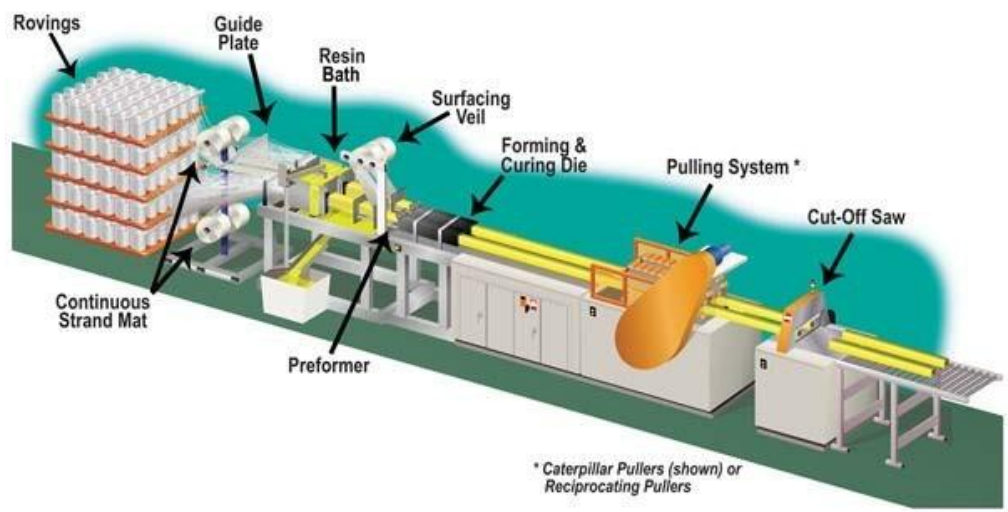

Fuente: Strongwell Corporation. 
El desarrollo de este tipo de materiales nace de la necesidad de tener productos resistentes mecánicamente, resistentes a la corrosión, y de peso ligero. El perfil extruido de material compuesto es anisotropico con lo cual tenemos ciertas propiedades en diferentes direcciones del material. Una de las ventajas de este tipo de proceso en el producto final es que es ligero con respecto a los materiales tradicionales como el acero, aluminio y madera. Y tiene propiedades aislantes térmicas y eléctricas, así como un nulo mantenimiento. En conclusión el material obtenido tiene propiedades mejoradas respecto a sus componentes.

\section{Importancia del problema.}

En el campo de la extrusión de materiales compuestos es necesario controlar las variables del proceso, ya que de no hacerlo las propiedades físicas, mecánicas y químicas se verían afectadas de una manera decisiva.

Es por ello que se necesita predecir con anterioridad las características del nuevo material para que cumpla las exigencias en el campo que se va a desarrollar el perfil y las variables que ingresan en el proceso son de vital importancia para dicho fin. Este tipo de nuevos materiales obtenidos por medio de este proceso de manufactura en países de la unión Europea está ampliamente usado para diferentes áreas entre las cuales se está desarrollando con énfasis en el sector automotriz a través del proyecto COALINE, el cual lleva a cabo nuevos procesos de fabricación para reducir costos y que el perfil cumpla las exigencias para que sea utilizado en dicha área.

La importancia de dichos materiales radical en el uso que se esta llevando a reemplazar a las estructuras hechas con materiales tradicionales por este tipo de perfileria.

\section{Metodología.}

Como proceso de ingeniería, el diseño detallado transforma alternativas de conceptos, arquitecturas físicas preliminares, especificaciones de diseño y requisitos técnicos en definiciones de diseño finales e interdisciplinares. Estos diseños se ajustan y se elabora toda la documentación que les acompaña y que se necesita para fabricación con el fin de entregar producto que cumpla con los requerimientos deseados.

\section{Dosificación de la fibra de vidrio.}

Para tener características correctas del producto final en este caso un tubo cuadrado es importante que la dosificación de la fibra de vidrio tanto como su distribución dentro del 
molde sean importantes para que el material tenga las características deseadas, cabe decir que la sección es constante con lo que calculamos su área en la ecuación 1.

Figura 2. Dimensiones del perfil pultruido.

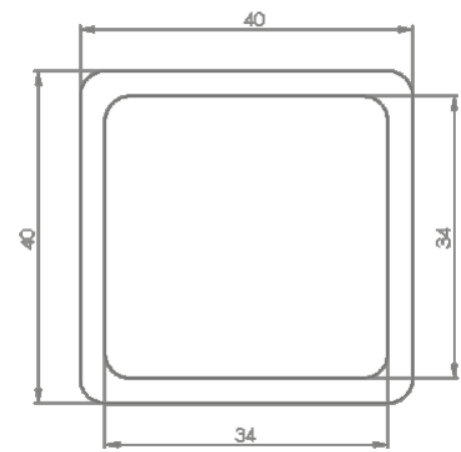

Elaborado por: Grupo de Investigación

En la ecuación 1 realizamos el cálculo del área del perfil a diseñar.

$$
\begin{aligned}
& \text { Area }_{\text {total }}=\text { Area }_{\text {externa }}-\text { Area }_{\text {interna }} \\
& \qquad \text { Area }_{\text {total }}=444 \mathrm{~mm}^{2}
\end{aligned}
$$

Recordando la parte teórica los moldes en este tipo de extrusión están estandarizados en un metro para lo cual vamos a calcular el volumen en ese apartado:

$$
\begin{aligned}
& \text { Volumen }_{\text {total }}=\text { longitud }_{\text {molde }} * \text { Area }_{\text {total }} \\
& \qquad \text { Volumen }_{\text {total }}=444000 \mathrm{~mm}^{3}
\end{aligned}
$$

La fracción del peso de la fibra de vidrio está en $65 \%$ y revestida de fibra mat, pero en este caso es necesario obtener algunos datos de la fibra de vidrio como es el Tex $(\mathrm{g} / \mathrm{m})$ que es el rendimiento en peso de la resina que es de 2400 Tex, cabe recalcar que el presente trabajo se realiza con hilo del fibra de vidrio grado $\mathrm{E}$. 
Las propiedades de la resina poliéster que provee a nivel Ecuador están indicados en la tabla 1. Como se puede observar son necesario las propiedades de la resina y la fibra de vidrio para obtener un valor correcto de las bobinar de hilo de fibra de vidrio.

Tabla 1. Propiedades de la resina isoftalica.

\begin{tabular}{llll}
\hline \multicolumn{4}{l}{ PROPIEDADES DE LA RESINA ENDURECIDA SIN CARGA (VALORES TIPICOS) } \\
\hline Propiedades & Valor & Unidad & Método ensayo \\
Densidad $\left(20{ }^{\circ} \mathrm{C}\right)$ & 1,21 & $\mathrm{~g} / \mathrm{cm}$ & DIN 53479 \\
Resistencia a la tracción & 70 & $\mathrm{MPa}$ & DIN 53455 \\
Módulo de elasticidad en tracción & 4300 & $\mathrm{MPa}$ & DIN 53 457 \\
Elongación a la ruptura & 2 & $\%$ & DIN 53 455 \\
Resistencia a la flexión & 110 & $\mathrm{MPa}$ & DIN 53 452 \\
Módulo de elasticidad en flexión & 4300 & $\mathrm{MPa}$ & DIN 53 457 \\
Temperatura de distorsión por calor (HDT) & 66 & ${ }^{\circ} \mathrm{C}$ & DIN EN ISO 75 \\
& 67 & ${ }^{\circ} \mathrm{C}$ & ASTM D 648 \\
\hline
\end{tabular}

Fuente: Adaptado datos del fabricante 2018.

Se hace la suposición que el perfil tiene el 100\% de su estructura compuesta únicamente de resina endurecida, pues sería el escenario donde su peso seria el máximo, entonces se tiene que en la ecuación 3:

$$
\begin{gathered}
\text { Masa }=\text { Volumen } * \text { Densidad } \\
\text { masa }=537.24 \mathrm{~g}
\end{gathered}
$$

Como se considera una relación de peso del $65 \%$ se tiene:

$$
\begin{aligned}
& \text { masa }_{\text {fibra }}=\text { masa }_{\text {resina }} * \% \text { en peso } \\
& \qquad \text { masa }_{\text {fibra }}=537.24 \mathrm{~g} \times 0.65 \\
& \text { masa }_{\text {fibra }}=349.2 \mathrm{~g}
\end{aligned}
$$

Para el cálculo del número de hilos de fibra de vidrio tenemos la siguiente razón que reúne la masa del perfil y el rendimiento de la fibra.

$$
\# \text { hilos }=\frac{\text { masa }_{\text {fibra }}}{T_{E X} \text { fibra }}
$$




$$
\begin{gathered}
\text { \#hilos }=\frac{349.2 \frac{\mathrm{g}}{\mathrm{m}}}{2.4 \frac{\mathrm{g}}{\mathrm{m}}} \\
\text { \#hilos }=145.5
\end{gathered}
$$

Para nuestro perfil tenemos 146 bobinas las cuales vamos a distribuir de la siguiente manera dentro del área de la sección transversal.

Figura 3. Disposición de hilos dentro de la sección transversal.

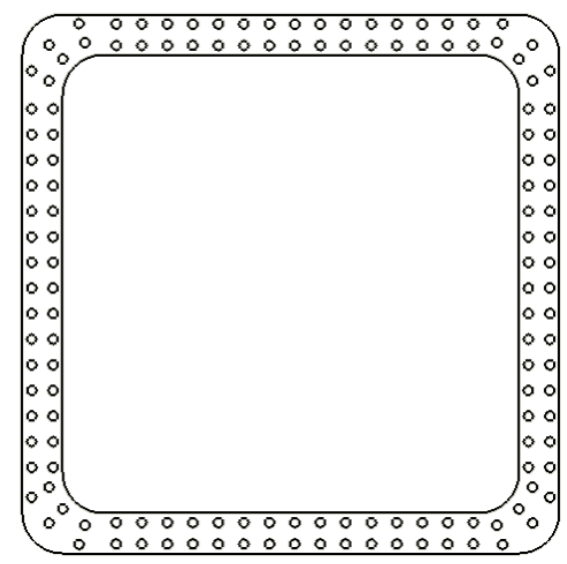

Elaborado por: Grupo de Investigación

\section{Tanque de resina.}

Otro de los puntos importantes de este proceso de fabricación es el tanque que contiene la resina y todo sus aditivos ya es esencial que disponga del volumen necesario para que el proceso pueda fluir sin ninguna interrupción. Es necesario calcular el volumen necesario para la fabricación de un metro de resina lo que esta expresado en la ecuación 6 es importante notar que el porcentaje en peso de la resina isoftalica en el perfil es del $35 \%$.

Vol $_{\text {resina }}=\frac{\text { Vol }_{\text {total }} \times 35 \%}{1 m}$

$$
\begin{aligned}
V o l_{\text {resina }} & =\frac{444 \mathrm{~cm}^{3} \times 35 \%}{1 \mathrm{~m}} \\
V o l_{\text {resina }} & =155.4 \frac{\mathrm{cm}^{3}}{\mathrm{~m}} \\
V o l_{\text {resina }} & =1.15 \frac{\text { litros }}{\mathrm{m}}
\end{aligned}
$$


Es muy importante tener en cuenta la cantidad de metros que se va a obtener de perfil por lo cual es necesario tener provista la cantidad de resina necesaria ya que un paro por falta de resina afectaría de una manera negativa las propiedades del elemento a estudiar.

\section{Molde y sistema de transferencia de calor.}

La ecuación de transferencia de calor dentro del molde en coordenadas cartesianas esta descrito en la ecuación 7:

$\rho C_{p}\left(\frac{\partial T}{\partial t}+V_{z} \frac{\partial T}{\partial z}\right)=k\left(\frac{\partial^{2} T}{\partial x^{2}}+\frac{\partial^{2} T}{\partial z^{2}}\right)+\rho \Delta H_{o} w_{r} R_{a}$

Donde $\rho$ es la densidad del compuesto, $\Lambda$ ho es el calor de la reacción del polímero, $\mathrm{W}$ res la fracción másica de la resina, $\mathrm{Ra}$ es la velocidad de reacción de la resina utilizada en este caso resina poliéster isoftalica, $\mathrm{Vz}$ es la velocidad de tirado de los hilos de fibra de vidrio. Con la ecuación 7 es necesario obtener la velocidad de la reacción de la polimerización (curado) que viene expresada en la ecuación 8 que involucra la exotermina.

$R_{r}(\alpha)=\frac{d \alpha}{d t}=k(T) f(a)$

Dónde $\alpha$ es el grado de cura, t es el tiempo de reacción, K es la el parámetro de temperatura de acuerdo a la ecuación de Arrhenius:

$K(T)=k_{O} \exp \left(-\frac{\Delta E}{R T}\right)$

Y

$f(\alpha)=(1-\alpha)^{n} g(\alpha)$

Dónde Ko es una contante, $\Delta E$ es la energía de activación, $\mathrm{T}$ es la temperatura absoluta, $\mathrm{R}$ la constante universal de los gases, $\mathrm{n}$ es el orden de reacción cinética, y $g(\alpha)$ es asumida para desarrollo de análisis como 1. La ecuación resultante es:

$R_{r}(\alpha)=\frac{d \alpha}{d t}=k_{O} \exp \left(-\frac{\Delta E}{R T}\right)(1-\alpha)^{n}$ 
En el proceso de extrusión de material compuesto, la concentración de la especie de resina en la matriz de formación se rige por la siguiente ecuación hiperbólica con fuente no lineal:

$\frac{d \alpha}{d t}=R_{r}(\alpha)-V_{z} \frac{\partial \alpha}{\partial z}$

Resolviendo las ecuaciones 12 y 7 obtenemos las temperaturas en las cuales los iniciadores para el proceso de polimerización son óptimos lo que nos impide que la polimerización se de en el tanque de resina. En la tabla 2 obtenemos dichas temperaturas con las que se trabaja.

Tabla 2. Zonas de calentamiento.

\begin{tabular}{llll}
\hline & Zona 1 & Zona 2 & Zona 3 \\
\hline Temperatura $\left({ }^{\circ} \mathbf{C}\right)$ & 70 & 120 & 170 \\
Longitud $(\mathbf{m m})$ & 280 & 220 & 200 \\
\hline
\end{tabular}

Fuente: Adaptado.

La transferencia de calor indicada en el molde se puede observar en la figura 10 con lo que se considera una temperatura ambiente de $20^{\circ} \mathrm{C}$.

Figura 4. Transferencia de calor en el molde.

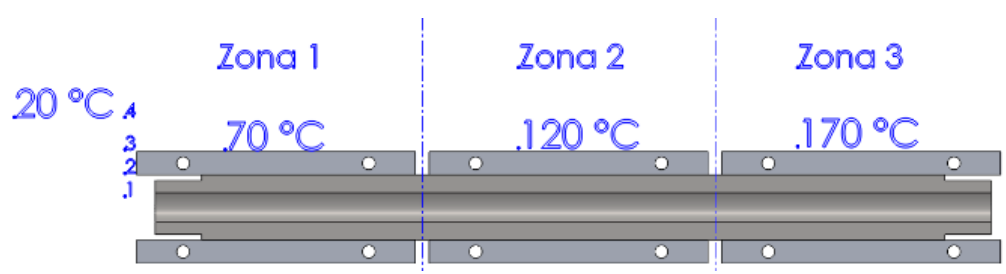

Elaborado por: Grupo de Investigación.

\section{Sistema de halado.}


Para el siguiente proceso de obtención del perfil se utilizó una máquina de extrusión de seis toneladas en el arrastre con lo que gracias a su sistema automatizado permite obtener velocidades de 0.05 a $0.4 \mathrm{~m} / \mathrm{min}$ por lo que va a permitir la adecuada velocidad para el curado. La máquina está provista de un sistema reciprocante. El corte de la perfileria se lo hace de manera manual con disco de widia

\section{Resultados.}

El número de bobinas para cubrir de una manera óptima a la sección transversal es de 146 por lo que a la disposición dentro del área es necesario distribuirlo de tal manera que pueda cubrir la mayor parte del área para no tener espacios en exceso entre los hilos con lo cual se cuenta ya con un diseño de un pre-conformador que se adapte de mejor manera al proceso. Otro de los datos para el proceso con el que contamos son las temperaturas ya que las ecuaciones que se tienen en el apartado anterior me proveen de las temperaturas necesarias para conseguir un curado recomendable estas temperaturas están en el molde.

Otra de las variables que al modificarla provoca cambios sustanciales en las propiedades del perfil que se está estudiando es la mezcla de la resina con sus aditivos. Una variación de sus iniciadores de polimerización cambia las temperaturas de trabajo porque a través de diferentes pruebas se tiene la siguiente concentración de la resina isoftalica y las concentraciones que los fabricantes recomiendan están expresada en los siguientes puntos.

Para la concentración de la mezcla:

- Resina poliéstres isoftalica la que va a ser nuestro estándar en porcentaje de peso es decir en $1000 \mathrm{~g}$

- Iniciador para temperatura baja Percadox 16 la concentración recomendada es 0.5 a $1.5 \%$.

- Iniciador TBPO la concentración recomendada es 0.5 a $0.25 \%$.

- Iniciador TBPB la concentración recomendada es 0.5 a $0.25 \%$.

- Pigmento Amarillo para resina poliéster 0.5-2.5\%

- Carbonato de calcio, se debe poner entre un $10-30 \%$.

- Talco industrial, las cantidad recomendad es $10-30 \%$.

- Alumina como agente desmoldante 0.5 a $1.5 \%$.

Para las zonas de calor:

- Zona 1: 60 - $80{ }^{\circ} \mathrm{C}$.

- Zona 2: $100-120^{\circ} \mathrm{C}$.

- Zona 3: $150-180{ }^{\circ} \mathrm{C}$.

Para la velocidad de arrastre de los pullers.

- Velocidad: 0,1 - 0,4 m/min. 
Después de realizar 10 pruebas se ajustaron los parámetros necesarios para poder obtener un perfil estable tanto dimensionalmente y sin contracciones.

Tabla 3. Parámetros óptimos para la obtención del Tubo.

\begin{tabular}{|c|c|c|c|c|c|c|c|}
\hline \multirow{2}{*}{ 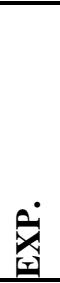 } & \multicolumn{7}{|c|}{ PARÁMETROS VARIABLES } \\
\hline & Preparación de & la resi & & $\begin{array}{l}\text { Ten } \\
\text { las } \\
\left({ }^{\circ} \mathrm{C}\right)\end{array}$ & $\begin{array}{l}\text { orat } \\
\text { onas }\end{array}$ & $\begin{array}{l}\text { is de } \\
\text { Calor }\end{array}$ & $\begin{array}{l}\text { Velocidad de tracción de los } \\
\text { pullers. }(\mathrm{m} / \mathrm{min})\end{array}$ \\
\hline \multirow{9}{*}{10} & Material & $\begin{array}{l}\text { Peso } \\
\text { (g) }\end{array}$ & $\%$ & Z1 & $\mathbf{Z 2}$ & $\mathbf{Z 3}$ & \\
\hline & Resina & 1000 & - & \multirow{8}{*}{70} & \multirow{8}{*}{120} & \multirow{8}{*}{170} & \multirow{8}{*}{0,15} \\
\hline & Iniciador P16 & 5 & 0,5 & & & & \\
\hline & Iniciador TBPO & 5 & 0.5 & & & & \\
\hline & Iniciador TBPB & 2.5 & 0.25 & & & & \\
\hline & Pigmento & 50 & 2 & & & & \\
\hline & Carbonato & 20 & 20 & & & & \\
\hline & Talco industrial & 10 & 10 & & & & \\
\hline & Alumina & 1 & 1 & & & & \\
\hline
\end{tabular}

Elaborado por: Grupo de Investigación.

\section{Discusión.}

Las propiedades de los materiales compuestos fabricados por extrusión dependen del contenido de fibra de vidrio, del tipo de resina y sus aditivos asi como de la fibra de vidrio que recubre exteriormente el perfil com lo es mat (velo) y su forma, es decir, la forma del entramado que pueden ser dispersas o tejidas. La resistencia del perfil depende en gran manera del contenido de fibra de vidrio con una conclusión que entre más fibra de vidrio tenemos más resistencia. 
El trabajo realizado en este trabajo es de un tubo cuadrado con fibras continuas de roving que están a lo largo del perfil como se observa en la figura 5 cabe recalcar que el material del perfil es de tipo "anisotrópico", esto significa que la resistencia no es la misma en todas sus direcciones, ya que es un perfil laminado que está compuesto de fibra unidireccional y resina poliéster.

Figura 5. Direccionamiento de hilos dentro de la sección transversal.

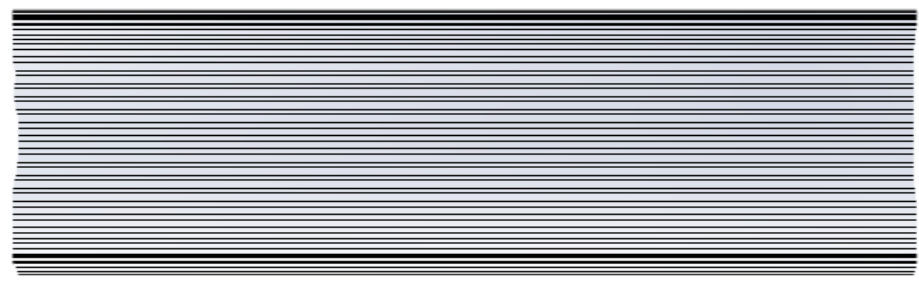

Elaborado por: Grupo de Investigación.

En la tabla 4, se muestra los valores referentes a tracción y flexión de cada proceso descrito y lo valores de trabajos anteriores.

Tabla 4. Propiedades mecánicas de varios procesos.

\begin{tabular}{llllllll}
\hline Proceso & Glass fiber $\%$ & $\boldsymbol{\rho} \frac{\boldsymbol{g} \boldsymbol{r}}{\boldsymbol{c m}^{3}}$ & $\begin{array}{l}\boldsymbol{\sigma} \\
\text { tracción } \\
(\boldsymbol{M P a})\end{array}$ & $\begin{array}{c}\text { Módulo tracción } \\
(\boldsymbol{G P a})\end{array}$ & $\begin{array}{l}\boldsymbol{\sigma} \\
\text { flexión } \\
(\boldsymbol{M P a})\end{array}$ & $\begin{array}{c}\text { Módulo flexión } \\
(\boldsymbol{G P a})\end{array}$ \\
\hline Filament wound & 80 & 2,07 & 551,58 & 27,57 & 689,47 & 34,47 \\
Moldeo compresión & 22 & 1,82 & 41,36 & 12,06 & 88,25 & 10,89 \\
Extrusión & 65 & 1,68 & 58,28 & 11,56 & - & - \\
Inyección & 22 & 0,18 & 33,78 & 10,55 & 87,56 & 9,92 \\
Hand lay-up & 30 & 1,35 & 86,18 & 6,89 & 193,05 & 5,17 \\
\hline
\end{tabular}

Elaborado por: Grupo de Investigación

En la tabla 5, se tiene los valores anteriormente obtenidos.

Tabla 5. Propiedades mecánicas.

\begin{tabular}{ll}
\hline & Mpa \\
\cline { 2 - 2 } modulo a flexión & 2.14 \\
esfuerzo a flexión & 46.23 \\
Módulo a Tesión & $11.56 \mathrm{Gpa}$ \\
Esfuerzo a Tensión & 58.28 \\
\hline
\end{tabular}

Elaborado por: Grupo de Investigación 
Ahora es necesario recordar los valores para el material pultruido que recomienda la EPTA (the European Pultrusion Technology Asocciation) cabe destacar que esta asociación reúne a los grandes productores de perfil de material comuesto a nivel mundial. La figura 6 tiene los valores recomendados y es necesario compararlo con los valores del material analizado.

Figura 6. Propiedades de materiales compuestos según la EPTA

\begin{tabular}{llc}
\hline Density & & $1,65-2,25 \mathrm{~g} / \mathrm{cm}^{2}$ \\
\hline Flexural strength & longitudinal & $44 / 500 \mathrm{~N} / \mathrm{mm}^{2}$ \\
Flexural strength & transversal & $200 / 300 \mathrm{~N} / \mathrm{mm}^{2}$ \\
Flexural modulus of elasticity & longitudinal & $17500 / 25000 \mathrm{~N} / \mathrm{mm}^{2}$ \\
Flexural modulus of elasticity & transversal & $12000 / 1700 \mathrm{~N} / \mathrm{mm}^{2}$ \\
Compression strength & transversal & $24 / 350 \mathrm{~N} / \mathrm{mm}^{2}$ \\
Tensile strength & longitudinal & $430 / 900 \mathrm{~N} / \mathrm{mm}^{2}$ \\
Tensile strength & transversal & $6 / 200 \mathrm{~N} / \mathrm{mm}^{2}$ \\
Elongation at break & longitudinal & $2,20 \%$ \\
Elongation at break & transversal & $1,60 \%$ \\
Coeffcient of expansion & & $1,5-5 * 106^{\Lambda}-\mathrm{xK}^{\Lambda}-1$ \\
Water absorption & & $1,16 / 2,5 \%$ \\
\hline
\end{tabular}

Elaborado por: Grupo de Investigación

Como se puede observar en la comparación de los valores que existe una diferencia en los valores recomendados por la EPTA, por lo que ellos utilizan procesos de producción de última tecnología así como distintos refuerzo e iniciadores por lo que estos valores son considerablemente altos.

\section{Conclusiones.}

Se tiene que las variables del proceso de pultrusión como es la disposición de los hilos, concentración de resina, las temperaturas en el molde, y la velocidad de arrastre del proceso son determinantes en la obtención del proceso. Necesario trabajar con los iniciadores corrector ya que se puede tener un plimerización prematura lo que daña absolutamente todo el proceso. Así como una velocidad alta produce burbujas de aire por donde puede crear fracturas en el material lo cual no es deseable. Para mayor resistencia en el perfil y aumentar las propiedades en las diferentes direcciones (recordar que estamos trabajando con un material anisotropico) es necesario recubrirlo con un velo o mat que puede tener la forma tejida o dispersa. El trabajar con fibras de hilo roving de grado $\mathrm{S}$ sería mejor ya que tendríamos mejores propiedades del elemento. Es importante ajustar el modelo para que 
pueda utilizarse con otro tipo de resinas por lo que para aplicaciones industriales de alta carga se utiliza una resina epoxica. La modelación matemática del proceso es necesaria para controlar de una manera recomendable las variables.

\section{Agradecimiento.}

Esta investigación no habría sido posible sin el apoyo de la empresa AISA COMPOSITES por lo que agradecemos la disposición por buscar mejoras tecnológicas por el bien de la sociedad.- Además este trabajo investigativo es el fruto del esfuerzo conjunto de conocimientos y experiencias laborales de los Ingenieros Fabián Sánchez, Marcelo Castillo, David Bravo, Patricio Abarca. Con estas líneas queremos mostrar nuestro agradecimiento a todas aquellas personas que supieron orientar nuestra investigación de una manera exitosa.

\section{Referencias bibliográficas.}

Sahuquillo Navarro, O. (2012). Estudio de la degradación de materiales compuestos base poliéster reforzados con fibra de vidrio en medios neutros y alcalinos. Valencia: Tesis doctoral - UPV.

Moschiar S. M.; Reboredo M. M.; Kenny J. M. and Vazquez A. Analysis of pultrusion processing of unsaturaed polyester resin with glass fibers, Polymer Composites, v17, n. 3, p 478, 1996.

Insausti Bello, A. (2007). Estabilidad global de elementos estructurales de polímero reforzado con fribra de vidrio. Donostia: Universidad de Navarra.

Moschiar S. M.;Santiago D.; Lombera G.; Urquiza S. Modelado numerico del proceso de pultrusion, Materials Research, Vol 6, No 4, 583-589, 2003.

Bendezú Reyes, J. (2002). Los plasticos reforzdos en fibra de vidrio (PRFV), sus aplicaciones y desarrollo en la industria nacional. Lima- Peru: Universidad nacional Mayor de San Marcos.

Liu X. L.; Crouch I.; Lam Y.; Simulation of heat transfer and cure in pultrusion with a general purpose finite element package, Composites Science and Technology 60 (2000) 857-864.

Calabrese L., V. A. (Noviembre, 2002). The effect of a liquid CTBN rubber modifier on the thermo-kinetic parameters of an epoxy resin during a pultrusión process. Composites science and technology, P6. 
Guerrero, V., Davila, J., \& Galeas, S. (2011). Nuevos materiales. Aplicaciones estructurales e indsutria. Quito, Ecuador: ImpreFepp.

Raymond W. Meyer, B. (1985). Handbook of pultrusion technology. New York: Chapman and Hall.

Kim D.; Lee W.; Friedrich K. A model for a thermoplastic pultrusion using commingles yarns, Compositees Sciense and Technology 61 (2001) 1065-1077 
Para citar el artículo indexado.

Sánchez E., Castillo E., Bravo V. \& Abarca E. (2018). Análisis del proceso de extruido de un tubo de material compuesto en resina isoftalica y fibra de vidrio. Revista electrónica Ciencia Digital 2(2), 334-347. Recuperado desde:

http://cienciadigital.org/revistacienciadigital2/index.php/CienciaDigital/article/view/104/97

\section{Ciencia}

El artículo que se publica es de exclusiva responsabilidad de los autores y no necesariamente reflejan el pensamiento de la Revista Ciencia Digital.

El articulo queda en propiedad de la revista y, por tanto, su publicación parcial y/o total en otro medio tiene que ser autorizado por el director de la Revista Ciencia Digital.
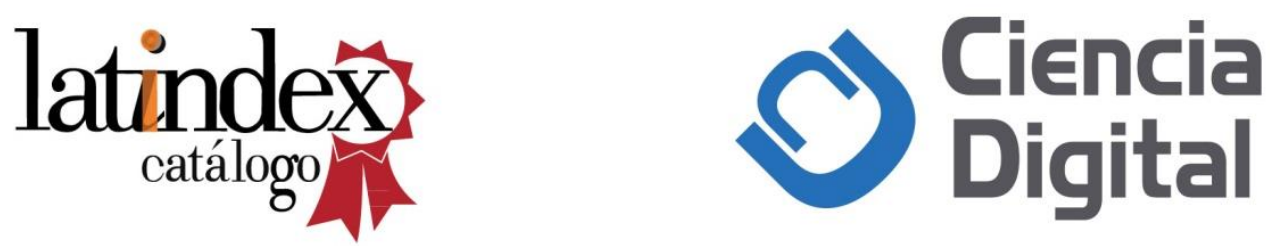